\title{
Practical considerations to achieve a strategic fit in the development of manufacturing ventures in developing countries
}

\author{
A.P. Brunet \\ Said Business School, Oxford University, UK \\ Merton College, Oxford OX1 4JD, UK \\ Tel. : +441844281621 \\ Fax. : +44 1844281316 \\ e-mail : pbrunetapb@aol.com
}

\begin{abstract}
The paper describes some of the opportunities and dangers of setting up a manufacturing operation with independent technological expertise in a developing country. Careful identification and review of the practical issues is advocated and illustrated with examples from a steel processing business established in Batam Island, Indonesia, the land of 'limited impossibilities' which now exports its services and products throughout the world.
\end{abstract}

Keywords

Manufacturing, Developing countries, Technical cooperation

\section{INTRODUCTION}

A company establishing local manufacturing capabilities in a developing country faces three key difficulties: the development of local staff, the identification and assimilation of suitable technology and obtaining the confidence of the market. These problems are compounded if there is no infrastructure to support 
manufacturing industry and if the company needs to export its products. In order to give such companies a chance to start their operations, many governments have established import substitution programs to protect local industry from the rigours of competition at the early stages of their growth. Although this practice is frowned upon by the world financial institutions since it can encourage companies to live in a cosseted world where they do not have to deal with real competition, realistically, it is the only way that a country can develop its own manufacturing capabilities against aggressive protective reaction from existing manufacturers. What is needed is a mechanism to ensure that after a period of establishment, companies are slowly exposed to external market forces. How can companies prepare for this?

The importance of a country maintaining its own manufacturing capabilities and not relying on its manufacturing industry being developed purely in the form of satellite operations of multi-nationals is highlighted by Buckley(1997) in their comprehensive review of international technology transfers by SMEs. Buckley found that such technical transfers provided an excellent opportunity for development and export in New World economies but that the technology providers found there was a shortage of companies with the independent ability to assimilate new technology under conditions where the principal company does not have the resources to provide long term and costly hand holding.

P.T. Citra Tubindo, an Indonesian processor of seamless steel tubes for the oil industry, has established a reputation of providing quality and reliability in an industry which is particularly sensitive to such matters and the company is now chosen by major steel producers to provide finishing services for their most demanding clients world-wide when the products require services which would disrupt their continuous manufacturing processes. This satisfactory outcome has only happened after many years of direct conflict with the integrated steel mills first in the domestic market and later in the regional and world markets and occurred due to the rational decisions taken in the choice of products and processes and steady development of the human resource capabilities.

The findings from the experience of Citra Tubindo should not be used as prescriptively but they serve to provide insight into the areas which should be considered by companies and individuals looking to develop manufacturing operations in the New World. In addition, the main message from Citra Tubindo's experience is fairly universal and should be the focus of every business plan. This is the need to choose options which provide an overall 'strategic fit' between the proposed technology and management systems and the environment in which they are to be deployed. Local advantages can be quickly eroded by poor fit. A square peg fitted into a round hole already leaves a gap indicating more than $20 \%$ surface loss and representing the environment as a much more complex hole serves to illustrate the huge potential loss of efficiency if a project of appropriate form is not inserted. Below I will consider some of the topics to be explored in establishing a fit which are relevant whatever the nature and type of business being considered. 


\section{MANAGEMENT SYSTEMS}

\section{The philosophy and vision}

\section{Time horizons}

A manufacturing venture in a developing country needs a clear vision of what it is trying to achieve. There are those to take advantage of short term local conditions and those which form part of an empire building exercise where the creation of lasting assets provides sufficient incentive. Is the plan for continued investment and growth? Can the company afford to take on liabilities for staff security? Can losses be made to establish market share or is immediate profitability more important? It may not be the specific choice of outlook which is important but a clear understanding of the desired direction. Even if the outlooks of the parties are different, a route to development can probably be found which accommodates each one but only if the intentions are clear. A de-facto leader among partners is needed to set the vision at any stage with the possibility of this leadership being handed over after a period of operations.

\section{Understanding the environment}

Whether a development under consideration is for a new local company or an investment from overseas, the operation needs to be tailored to suit the location and this requires complete understanding of the environment. Each location will offer a different balance of strengths and weaknesses. When considering a developing country the usual benefits relate to the cost of staff, availability of raw materials, cost of development and market opportunities but these potential areas of benefit may equally be the sources of the greatest problems. Is the low cost of staff balanced by a shortage of skills? An expatriate to compliment the skills of the local staff may cost the salaries of more than 100 workers causing great resentment as well as cost.

Is the infrastructure suitable for supporting the venture? It is better to ensure that a plan to provide all the needs is guaranteed before operations begin. The cost of standby generating power can be almost as much as providing continuous capacity. Are the formalities involved in import and export key to a project? Is this a possible source of problems? The cost of 'buying' effective passage through customs may affect the potential profitability of exports. The distances involved in local shipping may be short but they may be notoriously expensive and inefficient.

Can the project really get access to the local markets? Satisfying the customers is only half the battle. A protected market is a controlled one and local protection may be for the benefit of another local company. Even connections at the highest level cannot assure a place in the market. Co-operation with the lower level bureaucrats is often just as important as it is they who make the recommendations and paperwork for purchases unless the sums involved are very large. A venture can find itself in a no-man's land between the world market and the protected domestic market. 
Although an understanding of the local culture is important and there are certain taboos which need to be avoided, it is more often the practical considerations which dictate success.

\section{Development cycles}

\section{Government co-ordination and local competition}

Government protection may provide the only chance to start a national industry which can eventually compete in its own right. The steel industry is a good example of manufacturing industry where the level of proprietary knowledge and products does not exclude future outward development but reliance on multinational companies to develop the industry may preclude this. Examples of other industries include chemicals, fabricated goods, finished wood products and plastic products. In these industries there is the possibility of building a technology base within a new company through a combination of existing know-how, co-working with foreign companies, purchasing operating technology with the supply of equipment and steady internal development of operating techniques. The purpose of market protection should not be to allow the domestic prices to rise far above the global levels but rather to prevent the destruction of potentially competitive companies by overseas manufacturers eager to prevent newcomers into to their market. With the size of domestic markets usually limited and each manufacturer needing a critical volume, local competition can sometimes bring the price levels to below global levels but at least the market remains protected from irrational and political pricing effects. Once established, local companies must use the domestic market as a springboard to launch into external markets before new companies move in and grab their own territory.

\section{Attitude transformation to become a global player}

The move into global markets requires a complete change of attitude from the management and employees. There are no more second chances, orders have to be taken at price levels which challenge the plant staff and priorities have to be decided between servicing traditional clients and ensuring quality and reliability to convince new customers. This new pressure can lead to resentment which needs to be overcome by careful explanation. Cost levels must be achieved by maintaining high levels of capacity utilisation.

\section{Change from small to medium and large enterprise}

Another set of problems occur as the company grows in size. It may be possible to set out with an informal structure and communications system but as the company grows, more clearly defined channels have to be used to ensure the correct distribution of information and responsibilities. This process involves individual staff losing some areas of authority while enriching others, another source of resentment. Staff salary levels have to be aligned into a consistent system and reporting demands become greater for both internal and external monitoring. These extra layers of control add more costs which have to be countered by improvements through economies of scale, a concept which may not be well 
understood by the workforce. When Citra Tubindo merged two plants onto a new site, employees from both plants considered that the others were better treated. Careful handling of the personnel in such situations is necessary to avoid loss of effort due to misunderstandings.

\section{Foreign partnerships}

Co-operating with outsiders is almost inevitable at some stage in the company's development and the key to the success of such partnerships with foreign parties, be they for technical assistance or commercial co-operation, is that there must be logical benefits for all parties and between them there must be total coverage of the required capabilities. Too often parties underestimate the expectations of their partners. This can be avoided by looking at the problem from the perspective of each participant. If the logic is not there, whether the party realises it at the time of the agreement or not, then the partnership will eventually break down especially as the personalities involved will change with time and all the tacit agreements involved will be forgotten and only the written contracts remain. Once such logic is established then the distribution of benefits needs to be arranged to provide sufficient incentives for each party to maximise the joint performance and not just try to extract a better deal. It makes more sense to make a bigger cake than to take a bigger share.

Once the logic of a union is accepted then it is important to make sure that the scope of the contributions is clear and covers all the required areas. Firstly, the culture of the management systems must be established. Western management systems may work in a large project where financing is available to bring in a large contingent of expatriate staff to mimic operations in a developed country but this is expensive and fails to take advantage of the potential benefits following the national cultural systems. Ideally, in the case of a partnership of modest size, the local partner should provide the basis of the management system and structure for the manufacturing operations. Although they may seem to offer the best of both worlds, half and half solutions are the most difficult to implement as there are few staff whether local or expatriate able to create the elements of such a system and ensure an overlap rather gaping holes between the halves. With the framework of the management system resolved, the responsibilities and expectations of all parties must be fixed to avoid later misunderstandings. Once again it is important to make sure the arrangements encourage the partners to do the best for the project rather than wrangling over differences of opinion.

\section{CHOICE OF TECHNOLOGY}

\section{Finding the fit for products and processes}

Nowhere more than in choice of technology can a project be designed to succeed or fail. The choice of products and processes should be looked at together since the application of local advantages affects the process while the local market dictates the potential of the product so the two need to be balanced. 
Identification of strengths, weaknesses and aspirations

Each type of production and each environment present a different puzzle where there are no pieces which fit exactly. Only by having comprehensive understanding of the capabilities of the people, availability of assistance and service, nature of the market and the financial background and aspirations of the company can be best fit decision be made on the way to invest.

Poor level of service and support for equipment may disrupt even the best designed project even when the manufacturer has local representation. Despite being next door to Singapore, Citra Tubindo has often received better support from those companies operating directly from the USA, Europe or Japan than those with local support and in the end has had to develop independent maintenance capabilities.

Careful planning of infrastructure plays an important part in the success of operations but lack of available infrastructure is not necessarily a disadvantage. Citra Tubindo is able to generate its own power using cheap fuel and collect water from the local catchment ensuring low cost and no disruption to vital supplies.

The technological aspirations must be clear in the determination of the technical investment. A company intending to have complete mastery of its technology will do better to start simple and build up to more complex equipment and processes while only depending on technical assistance for the product concepts and design but maintaining control of the manufacturing processes. This provides for a slower start but in the end it will enable the company to produce any comparable products rather than being limited by the processes which match exactly to current products. The importance is often more in the power that comes with the knowledge than the commercial value of the knowledge itself since with complete control of the processes the company can bargain more equitably with its partners. A company with hybrid know-how can also attempt to spread its knowledge into similar markets as Citra Tubindo has done by establishing joint ventures in Malaysia, Brunei, Thailand and Vietnam succeeding where Japanese trading companies with more resources but less appropriate know-how have failed.

From the beginning there must be a commercial logic to the participation in the local market and for the expected moves beyond. Many questions need to be considered to establish this logic such as the following examples. Will the product be produced entirely by the company or only partially? Can the logistics costs of re-exporting be covered by the manufacturing cost advantage? How does the investment cost compare, on a per unit basis, with investments in developed countries? What is the status of world capacity for the products? Is there any potential for co-operation with other manufacturers to supply labour intensive services? Can the domestic market support the company in the short term?

\section{Nature of the investment}

Where labour costs are low, the choice of enabling technology should focus on improving certainty and reliability as opposed to extending output performance. In an environment such as Japan the increase of automation has not only reduced 
manpower costs but it has improved product reliability but in a developing country, the added maintenance demands are more likely to reduce reliability. Simple CNC machinery can eliminate the need for higher levels of operating skill while keeping maintenance and cost demands at a reasonable level and providing adequate product reliability. Surprisingly, there is little difficulty in training local staff to operate CNC equipment including simple offsetting, tool changing and set-ups.

Modular operating environments prevent complete production stoppages in the case of minor failures while providing more flexibility for product specialisation. This latter benefit can prove a major market advantage as has been the case in Citra Tubindo where integrated manufacturers have offloaded complex jobs at generous rates to avoid disrupting their own continuous production.

Establishing a market for an independent manufacturer even with some form of protection can take much longer than intended. Investment in modular technology based on simple concepts like those above allows for incremental growth not only easing pressure on cash flow but also on the development of human resources which must follow the growth of the company.

\section{Maintenance}

Maintenance has already been implicated as playing a part in the equation for deciding on the technology to use. The significance cannot be stressed strongly enough as even a perfectly designed plant beyond the maintenance capabilities of the environment is worth nothing and the transfer of maintenance know-how to local staff is extremely difficult.

\section{Technical transfer and the expected level of autonomy}

The technology likely to be required for manufacturing in a developing country is most likely available outside proprietary enclaves. On the other hand proprietary knowledge may provide valuable advantage in the marketplace. If the company intends to maintain its independence and develop its own technical know-how, it would do well to learn the manufacturing processes separately from co-operations to produce proprietary products. What is more, companies owning the rights to such propriety products may manufacture in a completely different environment and possess know-how for the actual manufacturing process which is not suitable.

\section{CHOICE OF PERSONNEL}

Labour costs are often a major justification for establishing manufacturing capabilities in a developing country but the disadvantages can sometimes outweigh the benefits. There is usually no shortage of candidates looking for work but their way of life has rarely prepared them for factory work. Lower level staff from developing countries are usually easy to manage and although they do not usually have skills or experience and are not used to working continuous sessions as in a factory, they will want to learn and adapt as well as possible. Some work concepts need to be introduced gradually. For example, overtime may not necessarily be productive, especially at the early stages. Workers on overtime may decide they 
have earned enough before the end of the month and just not turn up for a few days.

Finding management poses much greater problems. Although the response to management recruitment notices may be healthy, it is unusual to find candidates who truly fit the mould and the expected salaries are not the same bargains as the workers, especially if they are required to relocate. Many have apparently satisfactory paper qualifications but are not able to show the initiative required of a manager in a start up operation. On the other hand, there are others who will shine through from the most unlikely of sources and provide an opportunity to build an effective and loyal staff which should not be missed.

It can be the absence of any experienced staff at supervisory levels which causes the greatest difficulties. The lack of experience in the specific operations is no where near as serious as the lack of understanding the eight hour working day which is so different from village life where productive activities carry on at a modest pace but from dawn to dark. The pressure of maximising production is alien and to find candidates from the production line capable and willing not only to advise but also to pressurise their colleagues is a major problem and takes time and patience. Too much pressure will lead to an excuse culture and the workers will prove far more adept at producing excuses than the limited management will be at discrediting them!

A great benefit comes from the fact that the applicants for lower level jobs have not had any educational pre-selection so that in their midst is a broad spread of talent. It is this talent which must be sought out to provide the core for future management and to highlight the incentive that good work will be rewarded with improved opportunities. In Batam, there are strong laws protecting the jobs of permanent staff and a constant need to find new candidates for positions of responsibility. Since the company has a relatively high proportion of skilled and semi-skilled workers $(>65 \%)$, a policy has been established to only employ unskilled workers on short term contracts. If such workers are not able to convince the company of their ability to achieve the standard of operator by the end of the second contract then they are released to increase the chance of finding staff of higher ability from new candidates.

\section{Staff development}

The overseas package

The choice of timeframe and management system will determine what type of recruitment to perform. I will dwell longer on the local management variant but first note the success of many ventures primarily driven by a foreign partner, who has brought in a complete system and enough expatriate staff to be able to implement it and steadily train local staff to take over many of the jobs. Such a solution may satisfy certain priorities and in this case, the local market is not usually a significant factor. The oil business where the raw material rather than low prices are important or garment manufacturing and electrical assembly where low paid workers do very repetitive and easily organised jobs are examples of such opportunities. 


\section{Managing around available human resources}

Even for garment manufacturers it is now becoming common for local companies to operate as subcontractors with technical assistance to maximise the economy of local production. It is specifically this type of company operating its own systems and offering services as a subcontractor or providing its own products which offers the greatest opportunities from the local environment. The growing period for such companies is long and progress cannot be precisely planned in advance as it is dependent on the staff who eventually join leading to continual need for adaptation.

Recruitment is unpredictable and promised experience tends to be deceptive since those looking for new jobs have often performed under the wing of an experienced manager and been responsible for implementation rather than establishment of policy. Once exposed to the real responsibility of creative management such candidates are often ill prepared. Those with good potential have to be brought into the team and developed slowly. The strengths of some can be identified early on but those of others will only emerge later but still offer a critical resource. Japan's meteoric rise in manufacturing relied heavily on the high intelligence of many employees hired as workers who never had the chance to differentiate themselves in the higher education cycle. These staff are particularly key because not only do they provide the forces for improvement at grass roots level, but they are also economic and loyal since their experience is specific and they are not in a position to find other jobs easily.

Filling the position of Personnel Manager is perhaps the most difficult of all. The position is not identified as carrying a high level of prestige yet a successful candidate would need both an understanding of the local conditions and knowledge of more established human resource management techniques. The likelihood of finding the candidate is poor and the responsibilities tend to fall back on the General Manager or highest level national executive.

\section{Technical staff}

Technical staff are as rare as managers although hidden potential is perhaps easier to find. Our experience in Indonesia is that the country has a series of technical high schools which provide little more than a practical awareness but this is an adequate background for on the job development. Recruits at this level now perform most of our middle management and technical management functions. Staff recruited for technical jobs will usually readily accept to work and learn from others with superior knowledge whether local or expatriate and they find their own ways around the language barrier. It is important to note the poor ability to absorb information from classroom training. This appears to be an ability which is relatively alien and has not been successfully developed during schooling. On the other hand, reading abilities are adequate and the desire to learn often means that employees are prepared to spend their own time for self-development. 


\section{Use of expatriate staff}

The choice of expatriate staff is also difficult. More so if the company is a local venture taking on new technology or methods. Most foreign staff are used to working within a particular environment and while they may be experts in their fields, the majority tend to lack aptitude both to adjust their knowledge to a new environment and also to train personnel in the technology behind their skills. Due to these apparent difficulties, local staff learn by observation and only show their acquired knowledge when left to do the job themselves. It requires a leap of confidence to make the hand over from expatriate staff to locals but once it is done, progress is usually much faster than before. The earlier this leap is made, the better for the company in the long term, but one or two technicians with necessary patience and an understanding of the fundamentals, as opposed to detailed operational knowledge, should be retained to guide the local staff to find the solutions themselves. It is this learning by solving that leads to real understanding. This way of learning is particularly relevant to the development of maintenance teams as has been the case in Batam. Interestingly, the sphere of knowledge gained is limited and staff trained in this way have much less use to outside companies than their Western counterparts as has been discovered by the few operators and maintenance staff who have been tempted away by much higher salaries.

\section{Cultural isolation}

One disadvantage of building a company from the bottom up in the way illustrated above is that the culture becomes unique and even if suitable candidates can be found from outside to fill a position, they have great difficulty to fit into the culture. Many leave prematurely.

\section{Compensation}

The most important factor in compensation is to give the employees a sense of fairness and a feeling of opportunity to participate in the success of the company both through financial reward and job opportunity. A role in the company reflects the employee's position in the community and job security is a key motivating factor but is also a major liability to the company. At the initial stages, many employees will not be familiar with receiving a regular pay packet and care has to be taken to understand the implications of this. In developing communities, those who have employment or money are often expected to look after the welfare of other members of the family and community many of whom are free loaders. It is difficult to prescribe suitable welfare packages but any package should be devised to take such matters into account and protect the interests of the employee by providing food and welfare directly to him and his family as much as possible. As far as financial rewards are concerned, there is no benefit in paying salaries beyond the market level but bonuses can provide a useful incentive. The employees have their own ideas of how the company is doing and the periodic bonuses should reflect these more closely than actual performance. Assistance to the community and pastoral activities are also highly effective. 


\section{Developing innate Quality mindfulness}

One of the most difficult developments in the establishing a manufacturing operation in a developing country is to instil quality mindfulness. As with the position of Personnel Manager, the Quality Manager is not seen as having a prestigious position yet the transformation of attitudes to respect quality is paramount in importance and represents an alien concept to most workers. It is not so much the technical aspects of quality management which are difficult to obtain since these can be provided through outside assistance but such assistance is unlikely to be able to affect personal attitudes. It may become another of the major challenges and responsibilities of the General Manager to ensure discipline and respect for procedures using both carrot and stick. Procedures must be open to comments and suggestions from employees and there must be a simple way for quick change before procedures become disregarded or disrespected. Badly designed or impractical procedures will quickly lead to loss of control if they are not corrected since the workers will soon find their own ways of working around them. On the other hand a successful implementation of a quality program can lead to a level of excellence comparable to manufacturing plants anywhere in the world. Workers who are glad to have a job tend to be more diligent in repetitive activities such as inspection.

Wherever possible, it is better to avoid using consultants in the building of quality systems since they have a tendency to produce elegant rather than practical systems which never become an integral part of the operations.

\section{5}

\section{CONCLUSIONS}

As a combination lock cannot be opened until all the numbers have been correctly set, so a manufacturing venture in a developing country needs all parameters to be carefully established based on practical more than cultural considerations. Ignoring one of the variables in the equation may have little effect on the success of the project but it could bring it to a complete halt. Not all difficulties can be solved but there are few cases where they cannot be identified in advance.

\section{REFERENCES}

Buckley Peter J, Campos Jaime, Mirza Hafiz, White Eduardo, "International Technology Transfer by Small and Medium Enterprises", Macmillan (1997)

\section{BIOGRAPHY}

Paul Brunet is co-founder and chief executive of P.T. Citra Tubindo in Indonesia and is currently doing research in Operations Management at the Said Business School at the University of Oxford. 